\title{
The second and third waves in India: when will the pandemic be culminated?
}

\author{
C. Kavitha ${ }^{1}$, A. Gowrisankar ${ }^{1}$, Santo Banerjee ${ }^{2, a}$ \\ ${ }^{1}$ Department of Mathematics, School of Advanced Sciences, Vellore Institute of Technology, Vellore, \\ Tamil Nadu, India \\ 2 Department of Mathematical Sciences, Politecnico di Torino, Corso Duca degli Abruzzi 24, 10129 Torino, \\ Italy
}

Received: 11 May 2021 / Accepted: 18 May 2021

(C) The Author(s), under exclusive licence to Società Italiana di Fisica and Springer-Verlag GmbH Germany, part of Springer Nature 2021

\begin{abstract}
An unprecedented upsurge of COVID-19-positive cases and deaths is currently being witnessed across India. According to WHO, India reported an average of 3.9 lakhs of new cases during the first week of May 2021 which equals $47 \%$ of new cases reported globally and 276 daily cases per million population. In this letter, the concept of SIR and fractal interpolation models is applied to predict the number of positive cases in India by approximating the epidemic curve, where the epidemic curve denotes the two-dimensional graphical representation of COVID-19-positive cases in which the abscissa denotes the time, while the ordinate provides the number of positive cases. In order to estimate the epidemic curve, the fractal interpolation method is implemented on the prescribed data set. In particular, the vertical scaling factors of the fractal function are selected from the SIR model. The proposed fractal and SIR model can also be explored for the assessment and modeling of other epidemics to predict the transmission rate. This letter investigates the duration of the second and third waves in India, since the positive cases and death cases of COVID-19 in India have been highly increasing for the past few weeks, and India is in a midst of a catastrophizing second wave. The nation is recording more than 120 million cases of COVID19 , but pandemics are still concentrated in most states. In order to predict the forthcoming trend of the outbreaks, this study implements the SIR and fractal models on daily positive cases of COVID-19 in India and its provinces, namely Delhi, Karnataka, Tamil Nadu, Kerala and Maharashtra.
\end{abstract}

\section{Introduction}

The World Health Organization (WHO) received the report of unexplained lung fever for the population of patients from Wuhan, China at the end of December 2019. Within next ten days, WHO confirmed that the communicable agent was an RNA virus of the Corona viridine family, declared to be a closely related species of the virus [1]. This led to the outbreak of Severe Acute Respiratory Syndrome (SARS) in 2002-2004 and the Middle East Respiratory

This letter is dedicated to warriors of the coronavirus and who sacrificed their lives across India and globe.

a e-mail: santoban@gmail.com (corresponding author) 
Syndrome (MERS) in 2012; however, this is new virus. The virus appears to have transmitted to people from a creature host (in this occasion, a bat) by attaining some new transformations. Formally known as SARS-CoV-2 and informally the novel corona virus 2019 (COVID-19), this virus causes a whole new respiratory disease. The COVID-19 pandemic has informed us how important it is to build a scientific temperament and propagate scientific culture in order to tackle a pandemic of this scale. The knowledge gained in this way helps us to understand the fundamentals of disease dynamics and to make progress in treating and preventing it. The second wave started in September 2020, and it is much steeper than the first wave. This is a combination of a large number of asymptomatic people and more infectious variant of the virus, suitable for indoor people. It is also constantly infected with viruses. For example, according to data from the Centers for Disease Control and Prevention (CDC) in the USA, a large proportion of British strains detected during genome surveillance in Delhi have a 50\% higher infection rate. In the second wave, positive cases and death cases of COVID-19 in India have been skyrocketing for the past few weeks, and India is in a midst of a catastrophizing second wave. The nation is recording more than 120 million cases of COVID-19; however, pandemics are not still reduced in most states. The states of India are interconnected so there are increasing chances of the virus spreading from one person to another person when social distance goes down. This is why states such as Delhi, Karnataka, Tamil Nadu, Kerala, and Maharashtra are mostly affected by the second wave.

Other than human factors, the evolution of corona virus is among the significant reasons behind the subsequent waves. Researchers have identified various transformations in the SARS-CoV-2, the corona virus that causes COVID-19. It is necessary to gain a deeper understanding of mutant strains and their pathogenicity by performing genomic sequences of more samples. Similarly, predicting the underlying transmission of the outbreak and analyzing the viability of control measures are urgent in evaluating the prospects for proceeding with transmission in fresher areas. This requires following the course of the pandemic to have the option to anticipate its rise for a superior reaction. In order to predict the dynamics of virus spread, numerous research works on COVID-19 have recently been proposed [13-19]. The dynamic evolution of the virus spread during the first wave as well as the second wave is investigated by SIR model (see [20-25]). The fractal-based models for COVID-19 pandemic have recently been investigated $[8,9,14]$. In particular, the fractal interpolation with fixed scaling factor is explored in [8] and optimal scaling factor is described in [9]. Usually, the free parameters named scaling factors have a decisive influence on the pattern of the fractal interpolation functions. Hence, in this model, the linear fractal interpolation is applied to predict the transmission rate by choosing the scaling factor from the SIR model. That is, the vertical scaling factors of fractal interpolant are generated by the SIR model according to the evolution of the epidemic curve. Further, the effective reproduction number is assessed which gives the number of new cases produced by an infected individual among the healthy persons.

This letter is organized as follows: Sect. 2 describes the mathematical formulation of fractal interpolation method and SIR model. The results of transmission rate prediction, effective reproduction number obtained in the proposed method and their interpretation are elaborately discussed in Sect. 3. Further, peak period of the second and third waves in India is elucidated in Sect. 3. The concluding remarks of the this study are narrated in Sect. 4.

\section{Mathematical formulation and development of model}

The fractal interpolation method is discovered based on the iterated function system which was made by visualizing the object of the world as a fractal. The basic focus of interpolation 
is the prediction of an unknown function in a given range from its accessibility in some set of finite points, thereby connecting the discrete system with the continuous system. Moreover, the choice of vertical scaling factors in fractal interpolation method provides a finest interpolant. That is to say, fractal interpolation also generates a classical interpolation by taking all the scaling factors as zero, whereas choosing of large value (close to 1) of scaling factors provides irregularity in the interpolant. In other words, fractal interpolations are not only replacing a classical interpolation scheme, whereas a new one but with a more versatile scheme recuperates the classical method. On the other hand, various mathematical models have been applied in communicable disease epidemiology for understanding the dynamics of epidemics and predicting consequences, thereby executing the effective strategies to control the outbreak. Among all, the SIR model is one of the most commonly executed models which is the foundation for other models; peoples within a society move between three major sections, namely Susceptible, Infected and Recovered as a virus spreads from individual to another individual [20,22-25]. In order to provide the effective prediction of the epidemic curve, this study utilizes the infection rate as the scaling factors in the fractal interpolation method. With this note, hereinafter, let us discuss the mathematical background applied in this study. Interested readers are requested to visit [2,6-8,20,22-25].

\subsection{Fractal interpolation method}

The number of positive cases of COVID-19 can be considered as a time series $\left(t_{n}, p\left(t_{n}\right)\right)$, where $p\left(t_{n}\right)$ denotes the number of positive cases at the time $t_{n}$. Usually, in epidemic analysis, the positive cases are observed day-wise, so here time is considered as a day. Thus, in order to predict the value of $p\left(t_{n}\right)$ corresponding to $t_{n}$, this study applied the fractal interpolation method. Let a given set of interpolation data $\left\{\left(t_{n}, p\left(t_{n}\right)\right) \in\left[t_{1}, t_{N}\right] \times \mathbb{R}: n=1,2,3, \ldots, N\right\}$. The following process explains the construction of an iterated function system (IFS) in $\mathbb{R}^{2}$ such that its attractor is the graph of the function which passes through the given data. Let $I=\left[t_{1}, t_{N}\right], I_{n}=\left[x_{n}, x_{n+1}\right]$, for $n=1,2, \ldots, N-1$ and define $N-1$ contraction homeomorphisms $L_{n}: I \rightarrow I_{n}$ such that

$$
L_{n}\left(t_{1}\right)=x_{n}, L_{n}\left(t_{N}\right)=x_{n+1} .
$$

For a suitable compact set $X$ of $I \times \mathbb{R}$, let $F_{n}: X \rightarrow \mathbb{R}$ be the $N-1$ continuous mappings such that

$$
F_{n}\left(x_{1}, p\left(t_{1}\right)\right)=p\left(t_{n}\right), F_{n}\left(t_{N}, p\left(t_{N}\right)\right)=p\left(t_{n+1}\right),
$$

and for some $k_{n} \in(0,1), n \in\{1,2, \ldots, N-1\}, F_{n}$ is a contractions with respect to $p(t)$. For $n=1,2, \ldots, N-1$, define $f_{n}: K \rightarrow I_{n} \times \mathbb{R}$ by

$$
f_{n}(t, p(t))=\left(L_{n}(t), F_{n}(t, p(t))\right) .
$$

Define the iterated function system (IFS)

$$
\left\{X ; f_{n}: n=1,2, \ldots, N-1\right\} .
$$

Associated with the IFS (4), there is a set-valued map $W: \mathcal{H}(X) \rightarrow \mathcal{H}(X)$ defined by $W(B)=\bigcup_{n=1}^{N-1} f_{n}(B)$, for any $B \in \mathcal{H}(X)$, set of all non-empty compact subsets of $X$. The Hausdorff metric, $H_{d}(A, B)=\max \left\{\max _{a \in A} \min _{b \in B} d(a, b), \max _{b \in B} \min _{a \in A} d(b, a)\right\}$, completes $\mathcal{H}(X)$, since $X$ is complete. It is well known that there exists a metric $d$ on $\mathbb{R}^{2}$, equivalent to the Euclidean metric, with respect to $d, f_{n}, n=1,2, \ldots, N-1$ are contractions $[6,7]$. As a consequence, $W$ is contraction on $\mathcal{H}(X)$; hence, by the theory of IFS, $W$ has 
a unique compact set $G_{f}$ such that $G_{f}=W\left(G_{f}\right)$ where $G_{f}:=\{(t, f(t)): t \in I\}$ is the graph of a continuous function $f: I \rightarrow \mathbb{R}$ which passes through the given data; thus, $f\left(t_{n}\right)=f\left(p\left(t_{n}\right)\right)$ for $n=1, \ldots, N$. The continuous function $f$ whose graph is the attractor of an IFS (4) is known as the fractal interpolation function (FIF) associated with the given data set $\left\{\left(t_{n}, p\left(t_{n}\right)\right) \in I \times \mathbb{R}: n=1, \ldots, N\right\}$. In this study, the linear fractal interpolation function (FIF) is applied for estimating the epidemic curve. To generate linear FIF, let us consider the IFS $\left\{\left[t_{1}, t_{N}\right] \times \mathbb{R} ; f_{n}: n=1,2, \ldots, N-1\right\}$, where

$$
f_{n}\left(\begin{array}{l}
t \\
p
\end{array}\right)=\left(\begin{array}{cc}
a_{n} & 0 \\
c_{n} & \alpha_{n}
\end{array}\right)\left(\begin{array}{l}
t \\
p
\end{array}\right)+\left(\begin{array}{l}
b_{n} \\
d_{n}
\end{array}\right)
$$

for $n=1,2, \ldots, N-1$. It is known that $f_{n}$ maps the endpoints of given data, i.e., $\left(t_{1}, p\left(t_{1}\right)\right),\left(t_{N}, p\left(t_{N}\right)\right)$, to the endpoints of each subinterval, i.e., $\left(t_{n}, p\left(t_{n}\right)\right),\left(t_{n+1}, p\left(t_{n+1}\right)\right)$, respectively. Hence, the mapping $f_{n}$ has constrained as

$$
f_{n}\left(\begin{array}{c}
t_{1} \\
p\left(t_{1}\right)
\end{array}\right)=\left(\begin{array}{c}
t_{n} \\
p\left(t_{n}\right)
\end{array}\right) \text { and } f_{n}\left(\begin{array}{c}
t_{n} \\
p\left(t_{n}\right)
\end{array}\right)=\left(\begin{array}{c}
t_{n+1} \\
p\left(t_{n+1}\right)
\end{array}\right)
$$

for all $n=1,2, \ldots, N-1$. These constrains gives the following system of linear equation

$$
\begin{aligned}
& a_{n} t_{1}+b_{n}=t_{n} ; a_{n} t_{N}+b_{n}=t_{n+1} ; c_{n} t_{1}+\alpha_{n} p\left(t_{1}\right)+b_{n}=p\left(t_{n}\right) ; c_{n} t_{N} \\
& \quad+\alpha_{n} p\left(t_{N}\right)+b_{n}=p\left(t_{n+1}\right)
\end{aligned}
$$

for all $n=1,2, \ldots, N-1$. We say $\alpha=\left(\alpha_{n}\right)_{n=1}^{N-1}$ is a scale vector. If $\alpha_{n}$ is a free parameter in the above system of liner equation, then it gives a unique solution. Hence, $a_{n}, b_{n}, c_{n}, d_{n}$ can be uniquely determined by the following equations

$$
\begin{aligned}
a_{n} & =\frac{t_{n+1}-t_{n}}{t_{N}-t_{1}} ; \\
b_{n} & =\frac{t_{N} t_{n}-t_{1} t_{n+1}}{t_{N}-t_{1}} ; \\
c_{n} & =\frac{\left(p\left(t_{n+1}\right)-p\left(t_{n}\right)\right)-\alpha_{n}\left(p\left(t_{n}\right)-p\left(t_{1}\right)\right)}{t_{N}-t_{1}} \\
d_{n} & =\frac{\left(t_{N} p\left(t_{n}\right)-t_{1} p\left(t_{n+1}\right)\right)-\alpha_{n}\left(t_{N} p\left(t_{1}\right)-t_{1} p\left(t_{N}\right)\right)}{t_{N}-t_{1}} .
\end{aligned}
$$

The free parameters $\alpha_{n}$ have a decisive influence on the pattern of the fractal interpolation function generated by the IFS [6,7]. Hence, in this model, the linear fractal interpolation is applied to estimate the epidemic curve by choosing the scaling factor from the SIR model as explained in the next section.

\subsection{SIR model}

The Susceptible-Infected-Recovered (SIR) model is formulated for epidemiology models which present the terminology, notation, and standard outcomes. Epidemic model is utilized to portray rapid outbreaks that happen in less than one year, while the endemic model is utilized for examining infections over prolonged periods, during which there is a reestablishment of defenseless by births or recuperation from transitory immunity. The classic SIR model gives a natural premise to seeing more intricate epidemiology modeling results [2]. The model equations of SIR, where S, I, and R stand for the number of susceptible, infected, and removed individuals (either recovered or died), respectively, are defined as follows: 


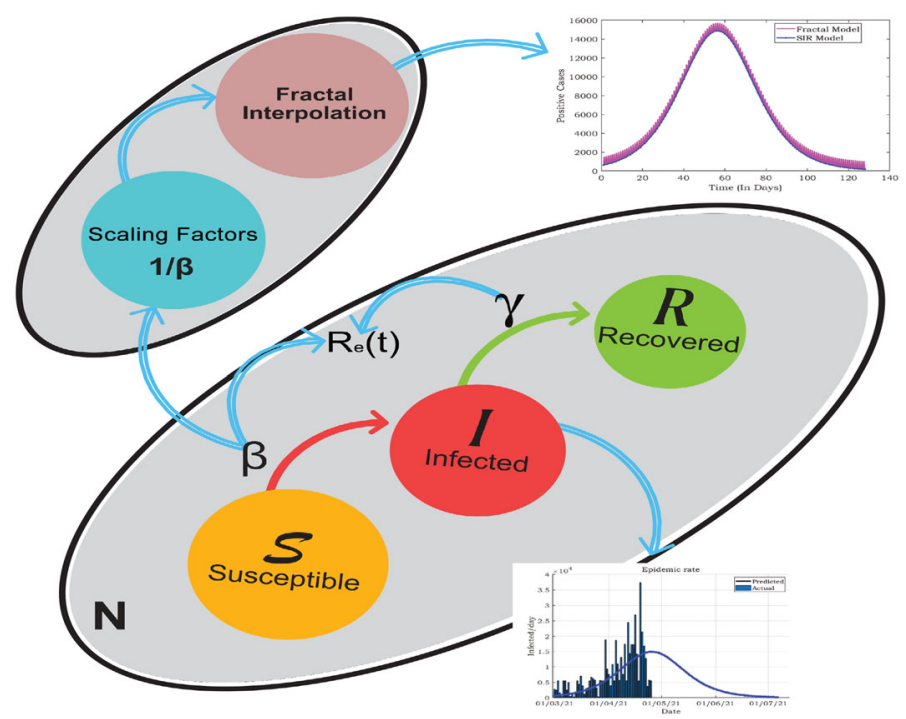

Fig. 1 The schematic diagram of the SIR and fractal model

$$
\begin{aligned}
\frac{d S}{d t} & =\frac{-\beta}{N} I S, \\
\frac{d I}{d t} & =\frac{\beta}{N} I S-\gamma I, \\
\frac{d R}{d t} & =\gamma I,
\end{aligned}
$$

where $t$ is the time, $N$ is the size of the total population, the number of susceptible individuals is denoted as $S(t)$, the number of infected individuals is given by $I(t)$, and the number of recovered individuals at time $t$ is symbolized as $R(t)$. Further, the estimation parameters $\beta, \gamma$ are the infection rate and recovery rate, respectively. In this work, the vertical scaling factors of the fractal interpolation function are obtained as the average infectious period from the SIR model to estimate the number of positive cases $p\left(t_{n}\right)$ at the time $t_{n}$. The process of proposed model is given in the schematic diagram Fig. 1.

\section{Results and discussion}

The data set of COVID-19 is obtained from the Indian government official website (https://www.mygov.in/covid-19/), and it is a data portal providing daily updated information of COVID-19 in India [12]. This data portal is developed and hosted by National Informatics Centre, Ministry of Electronics \& Information Technology, Government of India. This study deals the prediction of the transmission rate of COVID-19 based on fractal interpolation method and SIR model. In this study, we have selected the data set of daily positive cases of COVID-19 in most affected (more than 10 lakhs positive cases) five states of India, namely Delhi, Karnataka, Tamil Nadu, Kerala, and Maharashtra [12]. The data set has been accessed at the end of the day on April 30, 2021. More than a year has passed since the deadly corona virus infects people in India, and even after several efforts to control its spread, the second wave of the virus spread is now like a wildfire in the nation. As per the obtained data in [12], 


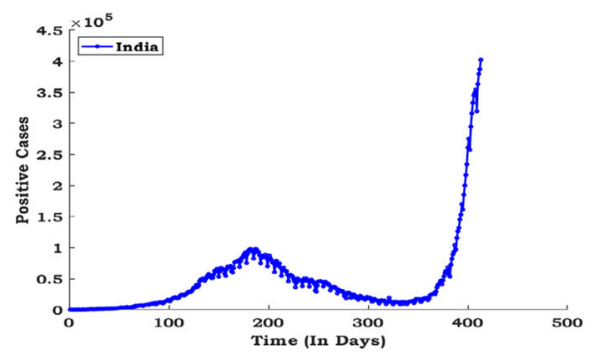

(a) India

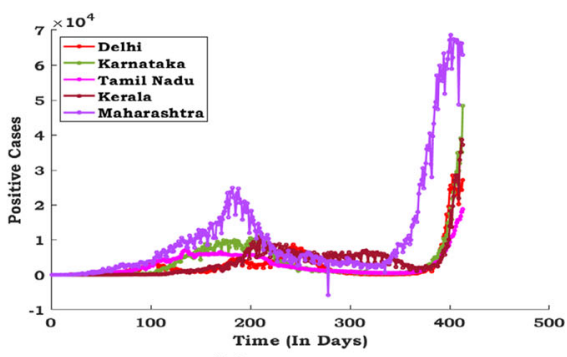

(b) States of India

Fig. 2 The evolution of the total number of positive cases as the epidemic curve from March 2020 to April 2021 for India and its states

India recorded 1,91,56,271 positive cases, the country with the second highest COVID-19 damaged across the world. Besides, Delhi, the capital of India, registered 11,49,333 cases, Karnataka reported 15,23,142 cases, Tamil Nadu showed 11,66,756 cases, Kerala attained $15,71,184$, and Maharashtra registered the highest positive cases in India as 46,02,472. The evolution of the total number of positive cases from March 14, 2020, to April 30, 2021, with respect to time is graphically displayed in Fig. 2. Observed from Fig. 2a, b, the second wave of corona spread started during the first week of March 2021 in India and its provinces.

The threshold measure for many epidemiology models is the reproduction number $R$, which is defined as the average number of secondary infections produced when one infected person is introduced into a host population where everybody is susceptible. The reproduction number $R$ is often considered as the threshold quantity that determines when an infection can attack and persist in a new host population [2]. Time-varying reproduction number can be calculated using time series of the infections and generation time distribution by Cori et al in [3]. In this study, effective reproduction number by the approach developed in [4] is obtained from the MATLAB software in which the MATLAB translation of $R$ function estimate_R estimates the reproduction number of an epidemic from the package EpiEstim [5]. The mean reproduction number is estimated for span of 7 days throughout the outbreak and $95 \%$ credible interval obtained from the incidence data. Also, we fixed the mean serial interval as 4.7 days with the standard deviation (SD) of the serial interval as 2.2 days. The evolution of mean effective reproduction number for 7 days from January 2021 to April 2021 is elucidated in Fig. 3, and India has $R$ value as 1.14. The reproductive number rate in prescribed states is as follows: $R=0.99$ in Delhi, $R=1.37$ in Karnataka, $R=1.26$ in Tamil Nadu, $R=1.32$ in Kerala, and $R=0.98$ in Maharashtra. The graphical representation of effective reproduction number $R$ of the prescribed states is demonstrated in Fig. 3b-f. The reproduction number is frequently measured in epidemiology as the threshold quantity that defines when an infection can invade and persist in new people. Even though positive cases are high at present time, from Fig. 3, it is observed that the reproduction number rate of Maharashtra and Delhi is less than 1; thus, the COVID-19 will decline and eventually expire in these two states. Karnataka, Kerala, and Tamil Nadu report the reproduction rate greater than 1, so the COVID-19 will be transmitted between people, and the outbreak will continue.

In this study, the epidemic curve is two-dimensional graphical representation of COVID19-positive cases, where abscissa corresponds to the time in days, while the ordinate presents the number of positive cases. In the literature of covid-19 prediction model [8], it is observed that the irregularity is presented in the epidemic curves which is resembling a fractal. Hence, fractal interpolation method is investigated to estimate or approximate the epidemic curve; 


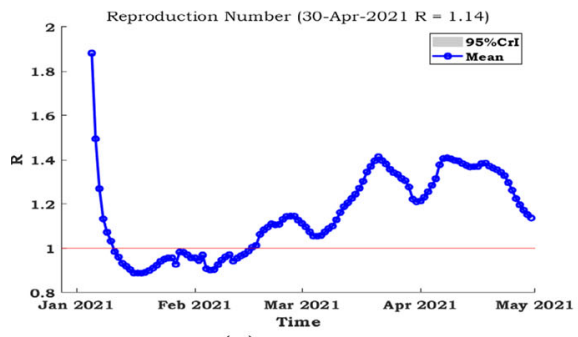

(a) India

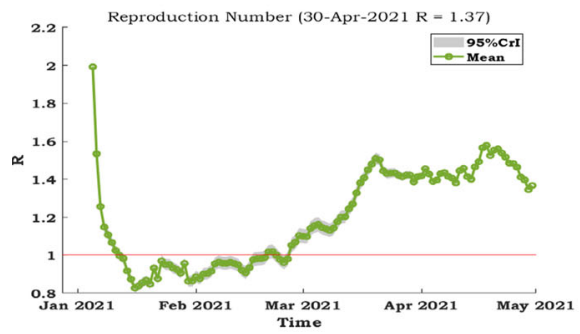

(c) Karnataka

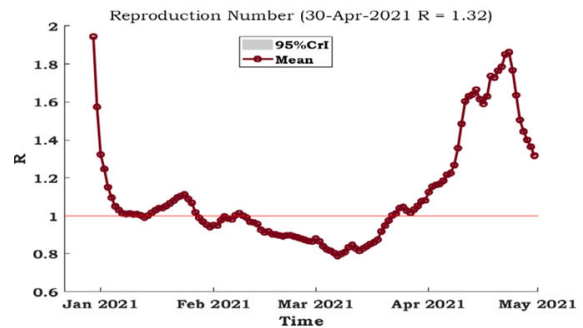

(e) Kerala

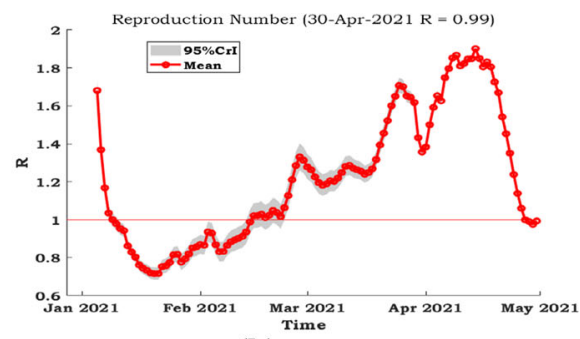

(b) Delhi

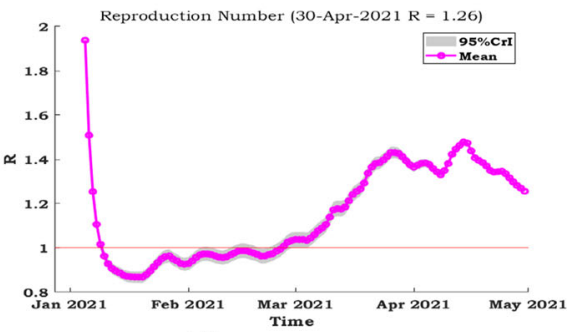

(d) Tamil Nadu

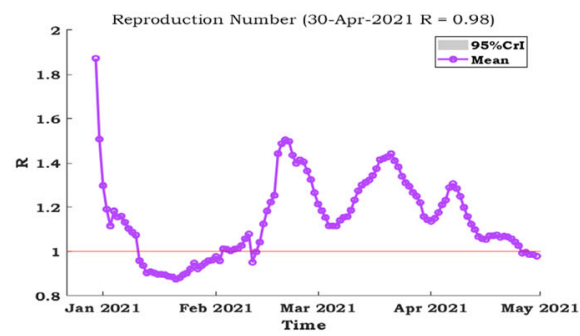

(f) Maharashtra

Fig. 3 The time evolution for the mean of reproduction number during the period January 2021 to April 2021 for India, Delhi, Karnataka, and Tamil Nadu

thereby, one can understand the dynamics of outbreaks. However, the choice of vertical scaling factor for fractal interpolation function is randomly fixed as 0.1 , thus scale vector also the same. In this proposed method, the fractal interpolation function with vertical scaling factors is obtained as the average infectious period from the SIR model to predict the epidemic rate (or infection rate) $p\left(t_{n}\right)$ at the time $t_{n}$. However, epidemic rate is the estimated number of new cases each COVID-positive case will infect. For instance, even though daily positive cases may be less, if the infection rate is high, then it indicates that daily positive cases will be high in the short future.

The SIR model is employed on the COVID-19 data set and approximated the progression of epidemic rate; usually, SIR model considered the positive cases (incidence) and recovery data to estimate the epidemic rate. The obtained results by Eq. 9 in the SIR model are described in the first column of Figs. 4, 5, 6 in which y-axis presents the number of infected persons per day. Similarly, the comparison between the predicted epidemic rate by fractal model and SIR is elucidated in the second column of Figs. 4, 5, 6. Here, the bell-shaped epidemic curve is approximated by choosing the scaling factors as the average infectious period in the fractal 


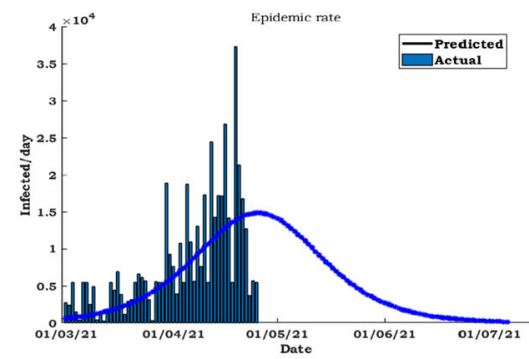

(a) India

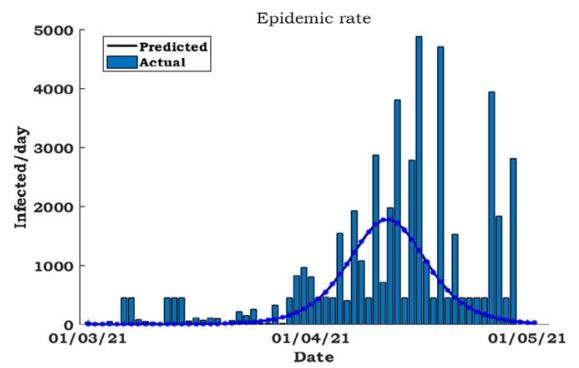

(c) Delhi

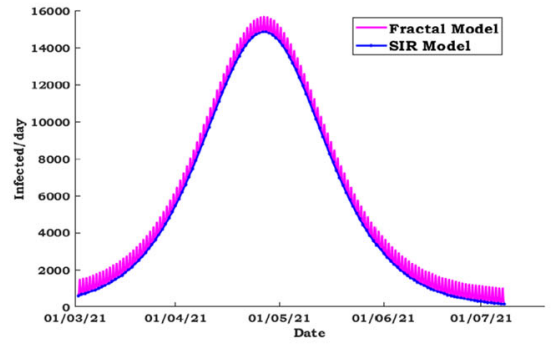

(b) India

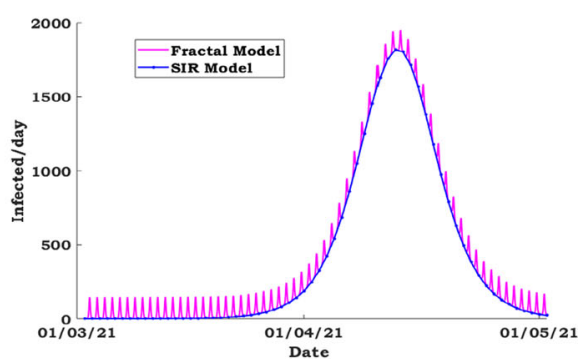

(d) Delhi

Fig. 4 Comparison of SIR model and fractal model in predictions of the COVID-19 epidemic. Prediction of epidemic rate: SIR model in the left frame and the fractal model in the right frame

interpolation function. Even though the coronavirus attacked the nation one year ago, the total number of positive cases was a peak in September 2020 and then gradually declined up to the end of February 2021, see Fig. 2a. Further, the second wave of the virus started in the first week of March 2021, and now it is staying like a wildfire in the nation. This study considered the number of positive cases from March 1, 2021, to April 30, 2021, to predict the forthcoming trend of the epidemic rate. As per the results displayed in Fig. 4a, b, both the SIR model and fractal model narrate that the second wave of the corona will be the peak in India during the mid-days of May 2021. Further, the eventually decreasing outbreak curve indicates that the growth of the number of positive cases significantly will reduce from the first week of July 2021 in India.

Besides, the SIR and fractal models fitted with the actual data on daily positive cases of COVID-19 in India and five provinces of India, namely Delhi, Karnataka, Tamil Nadu, Kerala, and Maharashtra. Practical data points are displayed as a bar plot, and the blue curve represents the predicted epidemic rate by SIR model; the magenta curve is the best fitting curve by the fractal model (refer Figs. 4, 6). Among the most impacted states of India, as per the predicted results, the capital Delhi and Maharashtra have already attained peak in the number of positive cases, see Figs. 4c, 6c. Meantime, Fig. 5 shows evidence that the southern state Karnataka is reporting the maximum number of positive cases and it would continue till the second week of May 2021, thereafter gradually decreasing, whereas neighboring southern state Tamil Nadu not yet registered its peak. As per the observed results in Figs. 5c, 6a by the proposed model, Tamil Nadu and Kerala will attain maximum from the second week of May 2021 to the end of May 2021. The reason for huge number of cases in prescribed states may be assembly election, because during the election period leaders and workers were unsuccessful to follow the corona protocol imposed by the government when organizing the political 


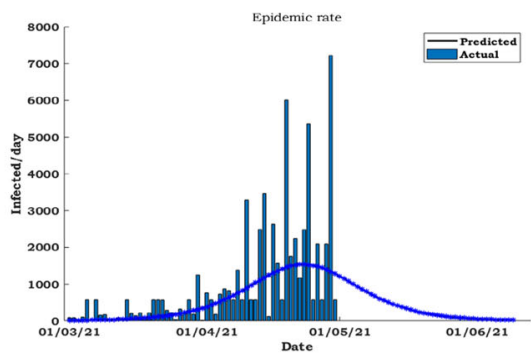

(a) Karnataka

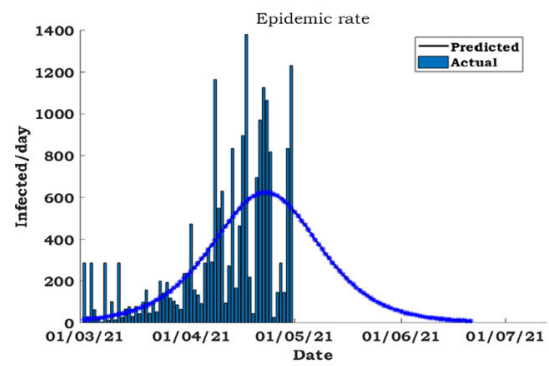

(c) Tamil Nadu

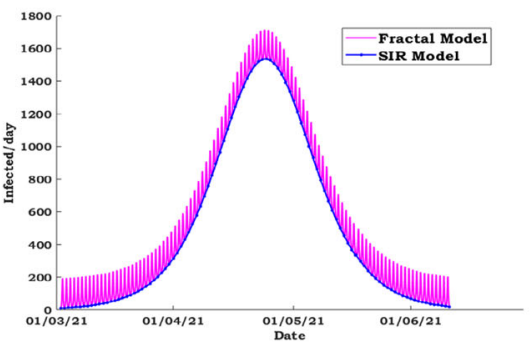

(b) Karnataka

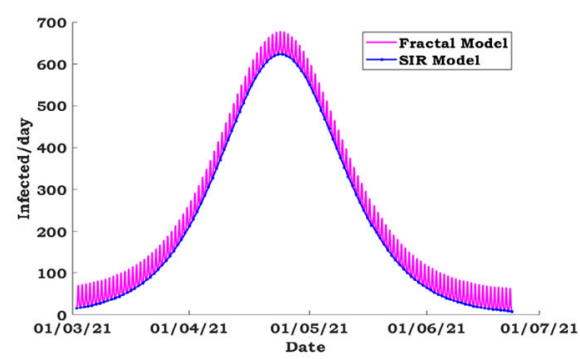

(d) Tamil Nadu

Fig. 5 Comparison of SIR model and fractal model in predictions of the COVID-19 epidemic. Prediction of epidemic rate: SIR model in the left frame and the fractal model in the right frame

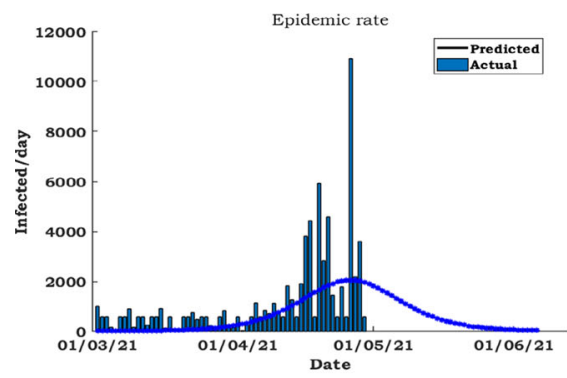

(a) Kerala

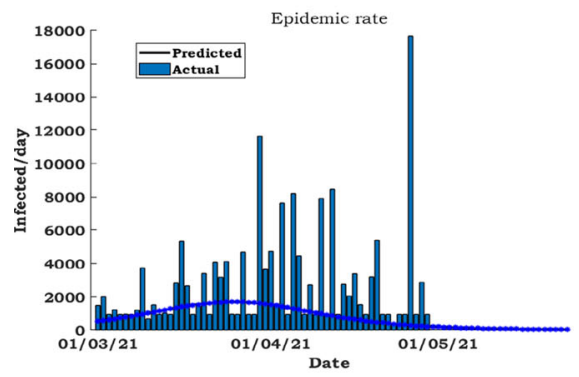

(c) Maharashtra

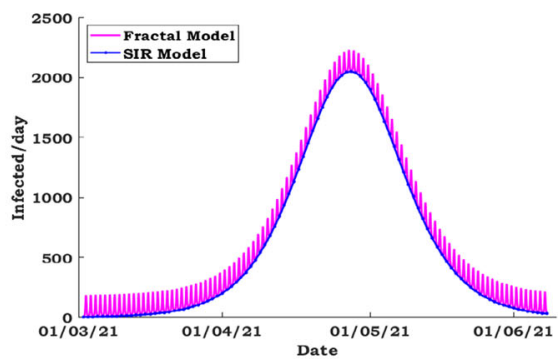

(b) Kerala

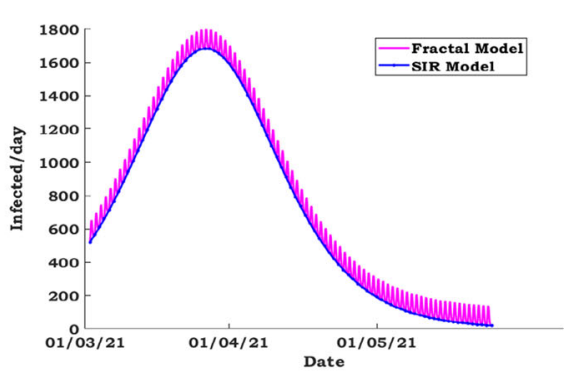

(d) Maharashtra

Fig. 6 Comparison of SIR model and fractal model in predictions of the COVID-19 epidemic. Prediction of epidemic rate: SIR model in the left frame and the fractal model in the right frame 


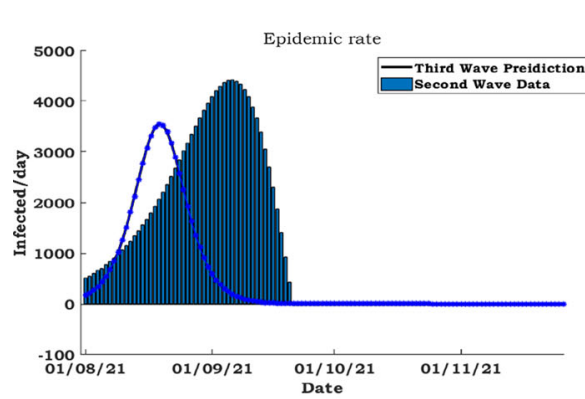

(a) India

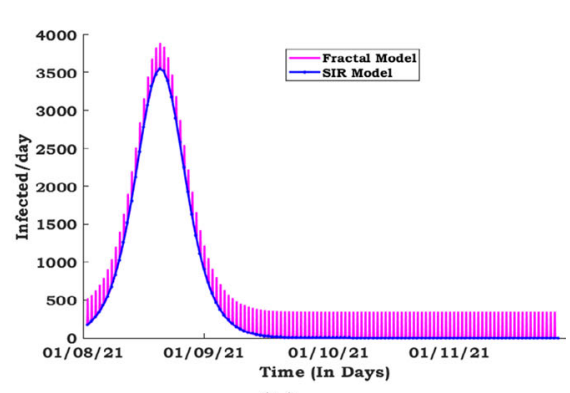

(b) India

Fig. 7 Third wave in India: prediction of the epidemic rate as the evolution of time

rallies. Further, a similar situation sent a confounding message to the general population and also the grassroot-level functionaries of the public authority. It weakened the vigil against the pandemic. As a result from Fig. 4a, b, it would be expected that the second wave of the corona will be in top on the mid of May 2021. Further, the outbreak curve eventually decays, and the growth of the number of positive cases significantly diminishes from the first week of July 2021. In Fig. 4b, compared with the SIR model, fractal model prediction narrates that the number of positive cases is declining but not fully vanished. Hence, this study suggests that the policymakers need to prepare for the likely arrangement and necessary actions. Moreover, if the second wave is not fully ruled out, then there is a possibility for the third wave. The next section discusses the third wave of COVID-19.

\subsection{Third wave}

Control measures implemented at the early stages during the first wave of COVID-19 helped in the successful mitigation of virus spread. However, the second wave started with unlocking social activities, so the positive cases during the second wave are rapidly increasing in India. According to the report of WHO, India reported an average of 3.9 lakhs of new cases during the first week of May 2021 accounting for $47 \%$ of new cases reported globally and 276 daily cases per million population. As per the results discussed in this study, the peak of the epidemic will occur in the second week of May 2021 and it will gradually decline from the first week of July 2021 in India. However, as per the discussion in the previous section, there is a possibility of a third wave in India. In order to predict the third wave of COVID-19, the predicted data of the second wave from the proposed model are considered as original data. The obtained outcome of the third wave is displayed in Fig. 7. Further, Fig. 7 shows that the third wave will start in the first week of August 2021 and will end during October 2021. As per our result in Fig. 7, the third wave will take a peak during the first week of September 2021 in India. Like the second wave, the predicted epidemic rate of the third wave by fractal model shows some peaks after October in Fig. 7. The third wave may be controlled by preventive arrangements, if so then these peaks will not occur. Here, one has to note that the proposed model depends on the available data and has not included the restrictive measures of COVID-19 such as lockdown and vaccinated people.

\section{Concluding remarks}

In overview, the proposed model analyzed the epidemic rate for the second and third waves of COVID-19 in India and five provinces of India, specifically Delhi, Karnataka, Tamil Nadu, 
Kerala, and Maharashtra. The obtained outcomes from the fractal and SIR models indicate that the peak of the epidemic will occur in the second week of May 2021 in India. Further, the eventually decreasing outbreak curve indicates that the growth of the number of positive cases significantly will reduce from the first week of July 2021 in India. In addition, the state-wise epidemic rate is discussed based on the results obtained by the SIR and fractal models fitted with the actual data on daily positive cases of COVID-19 in the second wave. In order to face and prevent the second wave, this study predicts the duration of peak weeks for the virus spread in India and its states which helps to prepare the possible arrangement by the policymakers. Besides, this study narrates that there is a possibility of a third wave in India, and it will start in the first week of August 2021 and will end during October 2021. The third wave will take a peak in the first week of September 2021. This study purely depends on the practical data available in the database as of April 2021, that is, some discussions and suggestions on the epidemic rate have been investigated based on the available data from the perspective of the proposed model. Further, the proposed model has not included the restrictive measures of COVID-19 such as lockdown, quarantine of suspected individuals, and their close contacts. As a consequence, the infection rate might eventually get decreased or increased accordingly with more information on the epidemic being available.

Acknowledgements Authors would like to thank Dr. Sanjay K Palit and Dr. Asit Saha for effective discussions toward the improvement of the article.

Availability of Data The data sets analyzed during the current study are publicly available.

\section{Declarations}

Conflict of interest The authors declare that they have no conflict of interest.

\section{References}

1. WHO, Coronavirus Disease (COVID-2019) Situation Reports, https://www.who.int/emergencies/ diseases/novel-coronavirus-2019/situation-reports/

2. H.W. Hethcote, The Mathematics of infectious diseases. SIAM Review 42(4), 599-653 (2000)

3. A. Cori, N.M. Ferguson, C. Fraser, S. Cauchemez, A new framework and software to estimate time-varying reproduction numbers during epidemics. Am. J. Epidemiol. 178, 1505-1512 (2013)

4. R.N. Thompson, J.E. Stockwin, R.D. van Gaalen, J.A. Polonsky, Z.N. Kamvar, P.A. Demarsh, E. Dahlqwist, S. Li, E. Miguel, T. Jombart, J. Lessler, S. Cauchemez, A. Cori, Improved inference of time-varying reproduction numbers during infectious disease outbreaks. Epidemics 29, 100356 (2019)

5. M. Batista, estimate_R (https://www.mathworks.com/matlabcentral/fileexchange/78760-estimate_r), MATLAB Central File Exchange. Retrieved May 2, 2021

6. S. Banerjee, D. Easwaramoorthy, A. Gowrisankar, Fractal Functions, Dimensions and Signal Analysis, Understanding Complex Systems, Springer (2021). https://doi.org/10.1007/978-3-030-62672-3

7. M.F. Barnsley, Fractal functions and interpolation. Construct. Approx. 2(1), 303-329 (1989)

8. P. Cristina-Maria, N. Bogdan-Radu, An analysis of COVID-19 spread based on fractal interpolation and fractal dimension. Chaos, Solitons Fractals 139, 110073 (2020)

9. D. Easwaramoorthy, A. Gowrisankar, A. Manimaran, S. Nandhini, B. Santo, R. Lamberto, An exploration of fractal based prognostic model and comparative analysis for second wave of COVID-19 diffusion, (2021) https://doi.org/10.21203/rs.3.rs-178464/v1

10. COVID-19 Data Repository by the Center for Systems Science and Engineering (CSSE) at Johns Hopkins University., https://github.com/CSSEGISandData/COVID-19

11. Roser, M., Ritchie, H., Ortiz-Ospina, E., Hasell, J., Coronavirus Pandemic (COVID-19), 2020, OurWorldInData.org, https://ourworldindata.org/coronavirus

12. National Informatics Centre, Ministry of Electronics and Information Technology, Government of India. https://www.mygov.in/covid-19 
13. M. Radiom, J.F. Berret, Common trends in the epidemic of covid-19 disease. Eur. J. Phys. Plus 135, 517 (2020)

14. A. Gowrisankar, R. Lamberto, S. Banerjee, Can India develop herd immunity against COVID-19? Eur. Phys. J. Plus 135, 526 (2020)

15. D. Rafiq, S.A. Suhail, M.A. Bazaz, Evaluation and prediction of COVID-19 in India: A case study of worst hit states. Chaos Solitons Fract. 139, 110014 (2020)

16. C. Pai, A. Bhaskar, V. Rawoot, Investigating the dynamics of COVID-19 pandemic in India under lockdown. Chaos Solitons Fract. 138, 109988 (2020)

17. P. Arora, H. Kumar, B.K. Panigrahi, Prediction and analysis of COVID-19 positive cases using deep learning models: A descriptive case study of India. Chaos, Solitons Fract. 139, 110017 (2020)

18. B.K. Sahoo, B.K. Sapra, A data driven epidemic model to analyse the lockdown effect and predict the course of COVID-19 progress in India. Chaos Solitons Fract. 139, 110034 (2020)

19. H. Seonga, H.J. Hyuna, J.G. Yun, Importance of early public health intervention, Comparison of the second and third waves of the COVID-19 pandemic in South Korea. Int. J. Infect. Dis. 104, 742-745 (2021)

20. Y.C. Chen, P.E. Lu, C.S. Chang, A Time-Dependent SIR model for COVID-19 with undetectable infected persons. IEEE Trans. Netw. Sci. Eng. 7(4), 3279-3294 (2020)

21. R. Ranjan Aryan, S. Mahendra, K. Verma, Characterization of the Second Wave of COVID-19 in India, Doi: https://doi.org/10.1101/2021.04.17.21255665

22. Mustafa Turkyilmazoglu, Explicit formulae for the peak time of an epidemic from the SIR model. Phys. D 422, 132902 (2021)

23. R. Schlickeiser, M. Kroger, Analytical solution of the SIR-model for the temporal evolution of epidemics: part B. Semi-time case. J. Phys. A Math. Theor. 54, 175601 (2021)

24. W.C. Roda, M.B. Varughese, D. Han, M.Y. Li, Why is it difficult to accurately predict the COVID-19 epidemic? Infect. Dis. Model. 5, 271-281 (2020)

25. N.M. Gatto, H. Schellhorn, Optimal control of the SIR model in the presence of transmission and treatment uncertainty. Math. Biosci. 333, 108539 (2021) 